\title{
THE EFFECTIVENESS OF INSERTION OF IMMEDIATE POSTPARTUM INTRAUTERINE CONTRACEPTIVE DEVICE (IPP-IUCD) IN TERMS OF MODES OF DELIVERY
}

\author{
Qudsia Qazi, Nazia Liaqat, Shehzadi Saima Hussain, Saima Khattak \\ Department of Gynaecology and Obstetrics, Lady Reading Hospital, Peshawar - Pakistan
}

\begin{abstract}
Objective: The objective of the study is to compare the effectiveness of IPP-IUCD in terms of mode of delivery

Methods: This observational study was conducted at Gynaecology and Obstetrics units of Lady Reading Hospital Peshawar of Khyber Pakhtunkhwa, Pakistan, where 200 patients, in whom postpartum IUCD was inserted within 10 minutes of delivery of placenta after normal vaginal delivery (group 1) or c/section (group 2), were included. These patients were grouped into 100 cases of vaginal and 100 cases of intra-cesarean IPP-IUCD insertions. Record of follow up of these 200 (19.9\%) patients was maintained and analyzed at 6 weeks to 12 weeks ` post-insertion follow up visits.
\end{abstract}

Results: No life-threatening complications such as perforation was recorded in both groups. Most common complications observed between vaginal vs intra-cesarean insertions were irregular vaginal bleeding in (11\% vs 5\%), abdominal pain (6\% vs $9 \%)$, vaginal discharge ( $8 \%$ vs $5 \%$ ), spontaneous expulsions ( $8 \%$ vs $2 \%)$ and lost strings (14\% vs $1 \%)$. Statistically significant difference was found between two groups with respect to lost strings $(P=0.0006)$.

Conclusion: IPP-IUCD is an acceptable, safe (in terms of complications) and effective contraceptive option after both vaginal and intra-cesarean insertions. Early follow up examinations are helpful in identifying spontaneous expulsions and dealing common problem.

Keywords: Immediate postpartum IUCD, mode of delivery

This article may be cited as: Qazi Q, Liaqat N, Hussain SS, Khattak S. The effectiveness of insertion of Immediate postpartum intrauterine contraceptive Device (IPP-IUCD) in terms of modes of delivery. J Med Sci 2020 October;28(4):367-371

\section{INTRODUCTION}

Pakistan is sixth populous country in the world, making child spacing an unmet need especially in the first year of childbirth. Undesired pregnancy in this period is mainly because sexual activity is resumed earlier and ovulation is unpredictable. ${ }^{1,2}$ Pakistani population was estimated as 207.77 million in 2018 , a growth rate of $1.89 \%$, one of the highest fertility rates in the region second only to Afghanistan, hampering socioeconomic development ${ }^{3,4}$. The prevalence of unintended pregnancy is $38-46 \%$ while that for use of modern methods of contraception is $26 \%$ in Pakistan $^{5}$. This in turn leads to increased rates of induced abortion, maternal morbidity and mortality. A study observed that nonuse of contraception in postpartum period leads to unplanned pregnancies in $86 \%$ women and this ends up in $88 \%$ of induced abortions ${ }^{6}$.

\section{Correspondence \\ Dr. Nazia Liaqat}

Assistant Professor

Department of Gynaecology and Obstetrics, Lady

Reading Hospital, Peshawar - Pakistan.

Email: kamrantnk@gmail.com

Cell: +92-333-9463467

Date received: $31-05-2020$

Date revised: $08-06-2020$

Date accepted: $15-12-2020$
Most of the women are not clear about contraceptive use in postpartum period. The ideal time to motivate patients for engagement in contraception is intra and postpartum period i.e. after exhaustion of pregnancy and labor. In Pakistan, facility based births are $52 \%$, rendering PPIUCD an effective and excellent choice in reducing population burden of low resource countries like ours. All these facts emphasize the importance of provision of contraception in this sensitive period. According to world health organization "postpartum family planning (PPFP) program focuses on prevention of unwanted and closely spaced pregnancies in the first 12 months following childbirth. ${ }^{7,8}$ The Cu T 380A (IPPIUCD) in terms of efficacy is on the top of contraceptive devices with a failure rate of less than one per 100 women in the first year of use ${ }^{9}$. It is coitus independent, easy to insert and does not affect breastfeeding. Both care provider and clients are available in the same setting, securing time and cost of interval IUD insertion. A survey conducted from 2015-16 in Pakistan showed the highest reported (334) IUD insertions in KP while contraceptive prevalence rate was $46 \%$ compared to $81.8 \%$ for Islamabad ${ }^{10}$. There are 160 million IUCD users worldwide i.e.14.3\% of contraception users, so it is most widely used amongst modern methods of contraception ${ }^{11}$, 12. However, high expulsion rates are reported by other 
The Effectiveness Of Insertion Of Immediate Postpartum Intrauterine Contraceptive Device (Ippiucd) In Terms Of Modes...

studies (10.4-16.4\%) ${ }^{13}$. PPIUCD is associated with primary complications like pregnancy and secondary ones as irregular vaginal bleeding, abdominal pain, infection, expulsion and uterine perforation. These risks can be minimized by using standard technique and improved insertors. This fact is supported by an extensive literature review of Canadian Contraceptive Consensus ${ }^{14}$.

The changes in menstrual bleeding pattern encountered after immediate PPIUCD are distressing for patient. Reassurance of patients with mild symptoms is sufficient i.e. mild changes are due to postpartum involuting uterus. Mild cramping pain can occur in the first few weeks of insertion. IUCD removal is an option for heavy and prolonged bleeding and constant abdominal pain. The upper genital tract infection risk is highest within first 15 to 20 days of insertion due to pre-existing infection and improper infection prevention practices ${ }^{15}$. Absent strings, most common in intra-cesarean insertion, at 6 weeks postpartum must be investigated via ultrasonography to ensure proper placement of IUCD. IPPIUCD counselling services regarding its common side effects and complications are important. Complications can be prevented by ensuring high quality training, follow up of training quality, infection prevention practices and training monitoring. Pros and cons of immediate postpartum contraception inserted via different routes need follow up in order to scale up PPIUCD program. There is minimal research comparing follow up outcomes between vaginal and caesarean insertions. This study is intended to compare outcomes of IPP-IUCD as a factor of route of insertion. The information gained will be utilized to provide evidence based counseling of the clients and to help them make informed choice regarding use of PPIUCD.

\section{MATERIAL AND METHODS}

This observational study was conducted in the Gynecology and Obstetrics department of Lady reading Hospital Peshawar that is a tertiary care hospital of the KP province of Pakistan, from October 2018 to September 2019, with two follow-up visits scheduled at six weeks for all the rest of outcomes and 12 weeks for irregular bleeding outcome. Ethical approval was obtained from hospital ethical committee. All patients fulfilling the inclusion criteria delivered vaginally or via c/section were included in study (consecutive sampling) i.e. clients in whom IUCD was inserted immediately after delivery of placenta. Post-insertion counselling was done by the person who inserted the device. Patients were given dates of their follow up on discharge card. For all the clients who got discharged uneventfully, follow ups were scheduled at 6th and 12th weeks. Telephonic contact was made with clients who did not return to health facility for follow up visit. Two hundred postpartum patients with IPP-IUCD insertion were included in study. Patients were grouped into two categories $(A)$ : first 100 cases of PPIUCD insertion following vaginal de- liveries (B): first 100 cases of PPIUCD insertions, intra cesarean. Patients with post abortion PPIUCD insertion were excluded from study. At 6th week visit, information regarding primary follow up outcomes like abdominal pain, abnormal vaginal discharge, lost strings and spontaneous expulsion, as a factor of route of insertion, was gathered and recorded on a proforma. At 12th week information on menstrual irregularity was collected. Abdominal pain was defined as constant crampy ache in the lower abdomen in the absence of urinary or bowel problem and relieved with or requiring painkiller use more than twice a week. Menstrual irregularity was defined as unpredictable, unscheduled vaginal bleeding occurring beyond six weeks postpartum. Pelvic examination was performed for abnormal vaginal discharge indicative of infection (foul smelling yellowish discharge), strings (which were trimmed if long) and presence of IUCD. If no threads were visible, ultrasonography was done to check for presence of IUCD and confirmation of its location. In case of complications the women were given treatment and reassurance. Data was analyzed on SPSS version 20 and chi square tests were used for comparison of qualitative data. Probability value of $<0.05$ was considered significant.

\section{RESULTS}

A total of 6283 deliveries occurred in our unit and 1005 (15.9\%) women opted for PPIUCD insertion over study period i.e. about 156 (15.5\%) intra-cesarean and $849(84.4 \%)$ after vaginal delivery. First 100 cases of vaginal and first 100 intra-cesarean (total 200) insertions were included in study. Most women (61.8\%) belonged to age group of 25-35 years, with multipara, para 4 or more (62\%). Of 200 patients, 103 (51.5\%) women came physically for follow up at 6 and 12 weeks after delivery while telephonic contact was made with 97 (48.5\%) cases (Table 1). Most of the complications were seen in insertions after vaginal delivery than cesarean section. Table-2 shows details of complications associated with 2 groups of women. Ultrasound was done for all women with undescended strings to confirm intrauterine position. No unplanned pregnancy or uterine perforation was recorded during the study. Patients with irregular bleeding were reassured that it will settle in further 2-3 months and Mefenemic acid and Tranexamic acid was advised. Medical treatment was not effective in $4 \%$ of vaginal and $1 \%$ of intra-cesarean insertion group $(P=0.368)$ and so removal of IUCD was the result. Total 10 IUCDs were expelled out of which 3 (1.5\%) IUCDs had partial expulsion, with a loop in cervical os. These were counted in expulsion group and removed . IUCD was removed in $14(7 \%)$ cases, the most common reason was irregular vaginal bleeding in vaginal group and abdominal pain (2\%) in intra-cesarean insertion group. Two percent of vaginal and $1 \%$ of intra-cesarean group underwent removal due to excessive vaginal discharge. 
The Effectiveness Of Insertion Of Immediate Postpartum Intrauterine Contraceptive Device (Ippiucd) In Terms Of Modes...

Table 1: Demographic variables of patients

\begin{tabular}{|c|c|c|c|}
\hline Variables & Groups & Number & \%age \\
\hline \multirow{2}{*}{ Age (Yrs) } & $18-24$ & 36 & 18 \\
\cline { 2 - 4 } & $25-35$ & 123 & 61.8 \\
\cline { 2 - 4 } & $>35$ & 41 & 20.9 \\
\hline \multirow{2}{*}{ Parity } & P1- P2 & 23 & 11.5 \\
\cline { 2 - 4 } & P3-P4 & 103 & 51.5 \\
\cline { 2 - 4 } & PP5 & 74 & 37 \\
\hline \multirow{2}{*}{ Follow up } & Physical & 103 & 51.5 \\
\cline { 2 - 4 } & Telephonic & 97 & 48.5 \\
\hline
\end{tabular}

Table 2: Risk of complications with insertions after vaginal vs $\mathrm{C} / \mathrm{section}$

\begin{tabular}{|c|c|c|c|c|c|c|}
\hline Complaints & Vaginal delivery Group & \%age & Cesarean section Group & \%age & Total & P Value \\
\hline NO Complaints & 66 & 66.0 & 65 & 65.0 & 131 & 1.0 \\
\hline Abdominal pain & 6 & 6.0 & 9 & $9.0 \%$ & 15 & 0.6 \\
\hline Menstrual irregularity & 11 & 11.0 & 5 & 5.0 & 16 & 0.2 \\
\hline Vaginal discharge & 8 & 8.0 & 5 & 5.0 & 13 & 0.50 \\
\hline Expulsion & 8 & 8.0 & 2 & 2.0 & 10 & 0.1 \\
\hline Lost strings & 1 & 1 & 14 & 14 & 15 & 0.0006 \\
\hline
\end{tabular}

\section{DISCUSSION}

A WHO report suggests that birth spacing can prevent $32 \%$ of all maternal deaths and over one million deaths of children below 5 years, thus, achieving positive maternal health outcome irrespective of contraceptive method. ${ }^{7}$ Postpartum women have a higher acceptability for contraception due to post-delivery conception fear. Postpartum IUCD can reduce expenditure of subsequent pregnancy particularly in developing countries as reported in study by Rodreiguz et al ${ }^{16}$. In this study, most (62\%) clients were multipara as they suffer ill health more due to morbidity associated with repeated pregnancies, consistent with a Taiwan study with $57 \%$ clients having four or more children. ${ }^{17}$ Although statistically insignificant but higher number of women followed up after intra-caesarean insertions as compared to vaginal insertions, probably due to fear of complications.

In our study, the common discomforts with PPIUCD insertion were abdominal pain, irregular vaginal bleeding, abnormal vaginal discharge and lost strings. The symptoms of irregular vaginal bleeding, lower abdominal pain and abnormal vaginal discharge were not influenced by route of insertion i.e. no statistically significant difference was found between the two groups. Our results are comparable with those of Welkovic S et al and Urmila T reporting abdominal pain in $8 \%$ of vaginal vs $10 \%$ of cesarean insertions. ${ }^{18,19}$ Another study showed comparable results i.e. excessive and continuous bleeding in $2 \%$ of vaginal and $5 \%$ of intra-cesarean insertions, respectively. ${ }^{2}$

In the study, no statistically significant difference was found between the two groups regarding vaginal discharge and expulsion rate ${ }^{12}$. The increased expulsion risk after vaginal compared to cesarean deliveries of $8 \%$ vs $2 \%$ comparable to $6.7 \%$ vs $4.8 \%$ reported by Tayyiba. ${ }^{20}$ The reason could be widely open os after vaginal delivery. A study by Abhijit reported expulsion rate of $4 \%$ and $2 \%$ respectively $(p=0.651){ }^{2}$

All patients were examined for visibility of threads at follow-up visit. Threads were visible among $99 \%$ of vaginal insertion clients as compared to $84 \%$ of intra-cesarean clients at follow up visit of 6 weeks. Our findings were consistent with that of an Indian study i.e. strings visible, at the end of 6 weeks, in $81 \%$ of vaginal and $65 \%$ of intra-cesarean insertions ${ }^{2}$.

In the study, statistically significant difference ( $p=0.0006$ ) was found between the two groups regarding visibility of threads at 6 weeks of follow-up i.e. no threads visible in $14 \%$ of intra-cesarean insertions. Most of lost strings were confirmed by ultrasound, lying curled up in endocervical canal. The study results are consistent with those of Abhijit i.e. missing strings in $16 \%$ of vaginal and $30 \%$ of intra-cesarean insertions, a statistically significant difference $(p=0.028){ }^{2}$ In a study by Nelson $A$ et al strings were visible in all the 7 intra-cesarean insertions at follow up. ${ }^{21}$ 
The Effectiveness Of Insertion Of Immediate Postpartum Intrauterine Contraceptive Device (Ippiucd) In Terms Of Modes...

In the study, IUCD was removed in $7 \%$ of clients ( $p=0.028$ ) due to bleeding problems, abdominal pain, vaginal discharge and incomplete expulsion. Celen reported removal of IUCD in $6-8 \%$ of clients while Tayyiba W reported in $16.8 \%{ }^{13}$. In an Indian study, removal was reported in $9 \%$ of vaginal vs $5 \%$ of intra-cesarean insertions. ${ }^{18}$ In the study, IUCD continuation rate was $89.5 \%$ compared to $90.6 \%$ of that reported by Reetu. ${ }^{22}$

Although, this study adds additional knowledge to the existing indication of use of IUCD practices, but further large scale studies of this kind are needed to enhance our understanding about the complications of insertion of IUCD in immediate postpartum period.

\section{CONCLUSION}

Postpartum IUCD insertions, both vaginal and intra-cesarean, are efficacious for contraception and safe in terms of complications. The postpartum IUCD is associated with low rates of abdominal pain, irregular vaginal bleeding, vaginal discharge and expulsion. Strings of IUCD are less visible after cesarean section in comparison to vaginal insertions at follow-up.

Proper timing of insertion, trained service providers and fundal placement using long Kellys forceps are fundamental in reducing expulsions and other complications. Media awareness and effective counselling of women about benefits versus risks is required to make such programs effective.

\section{REFERENCES}

1. Cleland J, Bernstein S, Ezeh A, Faundes A, Glasier A, Innis J. Family planning: the unfinished agenda. Lancet, 2006;368:1810-27.

2. Abhijit Halder, M.S. Sowmya, Abhimannyu G, Popli B et al. A Prospective Study to Evaluate Vaginal Insertion and Intra-Cesarean Insertion of Post-Partum Intrauterine Contraceptive Device: J Obstet Gynaecol India, 2016 Feb; 66(1): 35-41.

3. Safwat A M, Momen AK, Shabaan OM, Hossam TS. Acceptability for the Use of Postpartum Intrauterine Contraceptive Devices.Assiut Experience, Med Princ Pract, 2003;12:170-5.

4. National institute of Population Studies (NIPS) [Pakistan] and ICF International, 2013 Pakistan Demographic and Health Survey 2012-13. Islamabad:Pakistan. Calverton, Maryland, USA:NIPS and ICF International. + economic survey of Pakistan, 2015-16.

5. Habib AM, Greenow RC, Nausheen S, Soofi BS, Sajid $M$, Bhutta $A Z$ et al. Prevalence and determinants of unintended pregnancies amongst women attending antenatal clinics in Pakistan. BMC Pregnancy and Childbirth,
2017;30:17:156.

6. Y-M. Huang, R, Merkatz, J.-Z, Kang et al. "Postpartum unintended pregnancy and contraception practice among rural-to-urban migrant women in Shangai," Contraception, 2012;8(66):731-738.

7. World Health Organization. Programming strategies for postpartum family planning. Geneva: WHO; 2013. http:// www.mchip.et/sites/default/files/postpartum family-planning.pdf. Accessed, April 24:2014.

8. Arrowsmith ME, Aicken CR, Saxena S, Majeed A. Strategies for improving the acceptability and acceptance of the copper intrauterine device. Cochrane Database Syst Rev, 2012;CD008896.

9. Post-partum IUCD reference manual; Family Planning Division Ministry of health and family welfare. Gov India. 2010;1:2.

10. Contraceptive Performance Report 2015-16. Government of Pakistan, Statistics Division Pakistan Bureau of Statistics, apr 2017.www.pbs.gov.pk

11. Arcangues, C. Worldwide use of intrauterine devices for contraception. Contraception, 2007;75(6):S2-S7.

12. Grimes D. A., Lopez L. M., Schulz K. F., Van Vliet H. A., Stanwood N. L. Immediate post-partum insertion of intrauterine devices. Cochrane Database of Systematic Reviews, 2010;(5)CD003036.

13. S. Celen, A, Suak, Y, Yildiz and N Danisman. "Immediate Postplacental insertion of an intrauterine contraceptive device during cesarean section," Contraception, 2012;84(3).240-42.

14. Black,A, Guilbert, E, Costescu, D, et al. Canadian contraception consensus (part 3 of 4): Chapter 7-intrauterine contraception. J Obstet Gynaecol Can,2016;38:182-222.

15. Immediate postpartum insertion of an IUD is safe and effective. Global Health Technical Briefs,2007. USA: USAID (United State Agency for International Development and Family Health International), 2007. Laproz Grimes,Szpir (Family Health International/CRTU Programme).

16. Rodriguez MI, Caughey AB, Edelman, Darney PD, Foster DG. Cost-benefit analysis of state - and hospital-funded postpartum intrauterine contraception at a university hospital for recent immigrants to the United States, Contraception,2010 Apr;81:304-8.

17. Joint monthly report, Republic of China,Taiwan Population Studies Centre, Taiwan Provincial Department of Health and Planned Parenthood Association, (july-August) 1968 .

18. Umrila Tripathi, Durga Sahu. Outcome of immediate postpartum insertion of IUCD- A prospective study. Indian Journal of Obst and Gynaecol Research,2018;5(4):511-515.

19. Welkovic S, Costa LO, Faúndes A et al. Post-partum 
The Effectiveness Of Insertion Of Immediate Postpartum Intrauterine Contraceptive Device (Ippiucd) In Terms Of Modes...

bleeding and infection after post-placental IUD insertion. Contraception, 2001;63:155-158.

20. Tayyiba W, Shysta S, Lubna J, Saima M. Outcome of immediate postpartum insertion of intrauterine contraceptive device: Experience at tertiary care hospital.Journal of Pakistan Medical Association, Apr 2018;68(4):1-13.

21. Nelson AL, Chen S, Eden R. Intraoperative placement of the copper T-380 intrauterine devices in women undergoing elective cesarean delivery: a pilot study. Contraception. 2009;80:81-83. doi: 10.1016/j.contraception.2009.

22. Reeto H, Sonika M, Smiti N, Anjali G, Hemant M et al. Immediate Postpartum Contraceptive Device Insertion in Cesarean and Vaginal Deliveries: A Comparative Study of Follow - Up Outcomes. Int J of Reprod Med, 2016; Article ID 7695847:5.
CONFLICT OF INTEREST: Authors declare no conflict of interest

GRANT SUPPORT AND FINANCIAL DISCLOSURE: NIL

\section{AUTHOR'S CONTRIBUTION}

Following authors have made substantial contributions to the manuscript as under

Qazi Q: $\quad$ Main idea, Data collection, Manuscript

Liaqat N: Writing of manuscript, Proofreading, data collection

Hussain SS: Data analysis, Proof reading

Khattak S: Manuscript writeup

Authors agree to be accountable for all aspects of the work in ensuring that questions related to the accuracy or integrity of any part of the work are appropriately investigated and resolved. 